\title{
Permeation of antigen protein-conjugated nanoparticles and live bacteria through microneedle-treated mouse skin
}

This article was published in the following Dove Press journal:

International Journal of Nanomedicine

20 June 2011

Number of times this article has been viewed

\section{Amit Kumar \\ Xinran Li \\ Michael A Sandoval \\ B Leticia Rodriguez \\ Brian R Sloat \\ Zhengrong Cui}

University of Texas at Austin, College of Pharmacy, Pharmaceutics Division, Austin, TX, USA
Correspondence: Zhengrong Cui University of Texas at Austin,

Dell Pediatric Research Institute,

I 400 Barbara Jordan Boulevard, Austin,

TX 78723, USA

$\mathrm{Tel}+\mathrm{I} 5 \mathrm{I} 24954758$

Fax + I $51247 \mid 7474$

Email zhengrong.cui@austin.utexas.edu
Background: The present study was designed to evaluate the extent to which pretreatment with microneedles can enhance skin permeation of nanoparticles in vitro and in vivo. Permeation of live bacteria, which are physically nanoparticles or microparticles, through mouse skin pretreated with microneedles was also studied to evaluate the potential risk of microbial infection.

Methods and results: It was found that pretreatment of mouse skin with microneedles allowed permeation of solid lipid nanoparticles, size $230 \mathrm{~nm}$, with ovalbumin conjugated on their surface. Transcutaneous immunization in a mouse skin area pretreated with microneedles with ovalbumin nanoparticles induced a stronger antiovalbumin antibody response than using ovalbumin alone. The dose of ovalbumin antigen determined whether microneedle-mediated transcutaneous immunization with ovalbumin nanoparticles induced a stronger immune response than subcutaneous injection of the same ovalbumin nanoparticles. Microneedle treatment permitted skin permeation of live Escherichia coli, but the extent of the permeation was not greater than that enabled by hypodermic injection.

Conclusion: Transcutaneous immunization on a microneedle-treated skin area with antigens carried by nanoparticles can potentially induce a strong immune response, and the risk of bacterial infection associated with microneedle treatment is no greater than that with a hypodermic injection.

Keywords: antibody responses, safety of microneedles, transepidermal water loss

\section{Introduction}

Microneedles have been researched extensively to improve intradermal or transdermal drug delivery. ${ }^{1-5}$ The feasibility of microneedle-mediated delivery of nanoparticles into or through the skin has also been confirmed. ${ }^{6,7}$ Initially, McAllister et al reported permeation of latex nanoparticles of up to $100 \mathrm{~nm}$ through human cadaver epidermis after the skin was treated with solid microneedles $(150 \mu \mathrm{m}$ long, base diameter $80 \mu \mathrm{m}){ }^{6}$ Coulman et al showed permeation of polystyrene nanoparticles $(138 \pm 25 \mathrm{~nm})$ through the micropores created in human skin by microneedles $280 \mu \mathrm{m}$ long with a base diameter of $200 \mu \mathrm{m} .^{7}$ In contrast, Zhang et al did not observe any permeation of poly(lactic-co-glycolic) acid nanoparticles $(166,206$, or $288 \mathrm{~nm})$ through human skin pretreated with microneedles $200 \mu \mathrm{m}$ long but did show penetration of nanoparticles into the epidermis and dermis. ${ }^{8}$

New-generation vaccines based on recombinant DNA technology generally need a vaccine adjuvant to be strongly immunogenic, and data from numerous studies have shown that many polymeric or solid lipid nanoparticles used as vaccine antigen 
carriers have potent adjuvant activity. ${ }^{9}$ One of the attractive applications of the combination of microneedle and nanoparticle technologies is in vaccine delivery. ${ }^{10}$ In fact, there have been significant and successful efforts to utilize solid microneedles coated with nanoparticle-based vaccine formulations, mainly virus-like particles, to perform transcutaneous immunization in animal models and in clinical trials. ${ }^{10-25}$ However, the feasibility of transcutaneous immunization by applying antigens carried by nanoparticles onto a skin area pretreated with microneedles has not been thoroughly evaluated. Although application of a vaccine formulation onto the skin prior to or after the skin area is treated with microneedles is associated with the slight inconvenience of being a two-step process, it does have some advantages. For example, the dose of vaccines that can be applied is not as limited as when the vaccine is to be coated on solid microneedles, and coating of a vaccine onto microneedles on a mass production scale is still a topic of active research. ${ }^{26}$ Recently, Bal et al showed that application of diphtheria toxoid formulated into nanoparticles $(211 \pm 4 \mathrm{~nm})$ prepared with N-trimethyl chitosan onto a mouse skin area pretreated with solid microneedles (300 $\mu \mathrm{m}$ long) induced an antidiphtheria toxoid antibody immune response. However, the response was not stronger than when the diphtheria toxoid was used alone. ${ }^{25}$ Interestingly, it was reported that the simple physical mixture of diphtheria toxoid with N-trimethyl chitosan nanoparticles was more immunogenic than diphtheria toxoid alone. ${ }^{25}$ Therefore, there continues to be a need to test whether transcutaneous immunization onto a skin area pretreated with microneedles with an antigen carried by nanoparticles is more effective than with the antigen alone.

Previously, Sloat et al reported the engineering of solid lipid nanoparticles of $200 \mathrm{~nm}$ in size from lecithin/glyceryl monostearate-in-water emulsions. ${ }^{27,28}$ It was shown that subcutaneous injection of protein antigens conjugated onto the nanoparticles induced strong functional antibody and cellular immune responses. ${ }^{27,29}$ In the present study, the antibody responses induced by ovalbumin nanoparticles or ovalbumin alone when applied onto a mouse skin area pretreated with microneedle rollers were evaluated and compared, using ovalbumin as a model antigen chemically conjugated onto nanoparticles (mean diameter $230 \mathrm{~nm}$ ) and three different microneedle rollers with different-sized microneedles. Prior to an in vivo animal immunization study, permeation of the ovalbumin nanoparticles through mouse skin treated with microneedle rollers was evaluated in vitro. Microneedle rollers are commercially available and used for cosmetic (self-application) and clinical treatment of the skin. It has been shown that sequential insertion of microneedles on a microneedle roller requires less insertional force than insertion of microneedles on a flat patch. ${ }^{2}$

Finally, a very important issue related to microneedlebased drug delivery has been rarely studied, ie, the potential risk of bacterial or viral infection via micropores created by the microneedles. Bacteria and viruses are physically nanoparticles or microparticles. Therefore, any micropores that allow the permeation of nanoparticles might also allow permeation of bacteria and viruses. It is generally assumed that the risk of infection associated with microneedle treatment is low, and many microneedle-related safety studies in clinical trials have focused on the degree of irritation and pain caused by the microneedles. ${ }^{30-33}$ The first study on the ability of microbes to traverse microneedle-induced micropores was reported by Donnelly et al, whereby permeation of microbes through porcine skin pretreated with a microneedle array $(280 \mu \mathrm{m}$ long, base diameter $250 \mu \mathrm{m}$ ) was confirmed in vitro. ${ }^{34}$ In the present study, an ex vivo model was designed to evaluate permeation of live bacteria through a mouse skin area pretreated with microneedles of different sizes. A nonpathogenic Escherichia coli DH5 $\alpha$ strain was used for this study.

\section{Materials and methods Materials}

Dermaroller $^{\circledR}$ microneedle rollers were purchased from Cynergy (Carson City, NV). Digital pictures of the microneedle roller are shown in Figures $1 \mathrm{~A}$ and 1B. There are 192 needles in eight rows on each roller. Three different microneedle rollers were used. Dimensions of microneedles on the different rollers are shown in Figure 1C. Based on the size of the microneedles, the microneedle rollers were named as rollers with large (1000 $\mu \mathrm{m}$ long, base diameter $80 \mu \mathrm{m})$, medium (500 $\mu \mathrm{m}$ long, base diameter $50 \mu \mathrm{m})$, and small (200 $\mu \mathrm{m}$ long, base diameter $20 \mu \mathrm{m})$ microneedles. Ovalbumin, fluorescein-5(6)-isothiocyanate, 2-iminothiolane (Traut's reagent), 3,3',5,5'-tetramethylbenzidine solution, sodium bicarbonate, sodium carbonate, Tween 20, and phosphate-buffered saline were from Sigma-Aldrich (St Louis, MO). Lecithin (soy, refined) was from Alfa Aesar (Ward Hill, MA). Glyceryl monostearate was from Gattefosse Corporation (Paramus, NJ). The 1,2-dipalmitoylsn-glycero-3-phosphoethanolamine-N-[4-(p-maleimidophyl) butyramide] (DPPE-maleimide) and 1,2-dioleoyl-sn-glycero3-phosphoethanolamine-N-carboxyfluorescein (DOPEfluorescein) were from Avanti Polar Lipids (Alabaster, AL). Goat antimouse immunoglobulin $\mathrm{G}$ was from Southern Biotechnology Associates Inc (Birmingham, AL). 


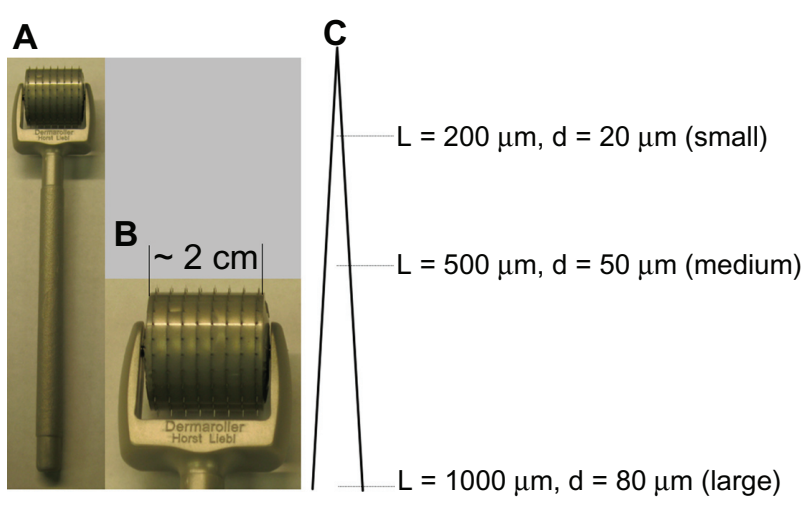

Figure I (A, B) Digital photos of a Dermaroller ${ }^{\circledR}$ microneedle roller $(1000 \mu \mathrm{m}$ long, base diameter $80 \mu \mathrm{m}$ ). (C) Diagram of microneedles on the three different Dermaroller microneedle rollers used (not to scale).

Note: $L$ indicates the length of the microneedles and $d$ is the base diameter of the microneedles.

\section{Preparation of nanoparticles}

Nanoparticles were prepared as previously described. ${ }^{27,28}$ Briefly, soy lecithin $3.5 \mathrm{mg}$ and glyceryl monostearate $0.5 \mathrm{mg}$ were placed into a $7 \mathrm{~mL}$ glass vial. One milliliter of deionized filtrated $(0.22 \mu \mathrm{m})$ water was added into the vial, followed by heating on a hot plate to $70-75^{\circ} \mathrm{C}$, with stirring and brief intermittent periods of sonication (Ultrasonic Cleaner Model 150T, VWR International, West Chester, PA). Once a homogeneous milky slurry was formed, Tween 20 was added in a stepwise manner to a final concentration of $1 \%$ $(\mathrm{v} / \mathrm{v})$ to form an emulsion, which was then allowed to stay at room temperature while stirring to form nanoparticles. The endotoxin level in the nanoparticle preparation was estimated to be $0.18-0.57 \mathrm{EU} / \mathrm{mL}$ using a ToxinSensor ${ }^{\mathrm{TM}}$ chromogenic limulus amebocyte lysate endotoxin assay kit from GenScript (Piscataway, NJ). ${ }^{29}$ The size and zeta potential of the nanoparticles were determined using a Malvern Zetasizer ${ }^{\circledR}$ Nano ZS (Westborough, MA).

To prepare the maleimide nanoparticles, DPPE maleimide, which has a reactive maleimide group, was included in the lipid mixture $(5 \%, w / w) .{ }^{27,29}$ To label the nanoparticles fluorescently, DOPE-fluorescein $(5 \%, \mathrm{~m} / \mathrm{m}$ of total lipids) was included in the lecithin and glyceryl monostearate mixture during nanoparticle preparation. ${ }^{27,29}$

\section{Conjugation of ovalbumin onto the nanoparticles}

The conjugation of ovalbumin onto the nanoparticles was completed as previously described. ${ }^{27-29}$ Prior to conjugation, ovalbumin was thiolated using Traut's reagent. Ovalbumin was diluted in carbonate buffer $(0.1 \mathrm{M}, \mathrm{pH} 9.6)$, followed by addition of Traut's reagent ( $20 \times$ molar excess) and a 60-minute incubation at room temperature. Thiolated ovalbumin was desalted using a PD10 column (GE Healthcare, Piscataway, NJ). To react the thiolated ovalbumin with the nanoparticles, $1 \mathrm{~mL}$ of freshly prepared maleimide nanoparticles were mixed with thiolated ovalbumin $(10 \mathrm{mg})$ in phosphate-buffered saline (0.1 M, pH 7.4) and stirred under nitrogen gas for 12 to 14 hours at room temperature. Unconjugated ovalbumin was removed by repeated ultracentrifugation $(600,000 \times \mathrm{g})$ and washing with phosphatebuffered saline three times. The amount of ovalbumin conjugated onto the nanoparticles was estimated as previously described using fluorescein-labeled ovalbumin..$^{27,29}$ Ovalbumin was labeled with fluorescein following the manufacturer's instructions (Promega Corporation, Madison, WI) before being conjugated onto the nanoparticles.

\section{Permeation of ovalbumin and ovalbumin nanoparticles through microneedle- treated skin}

An in vitro permeation assay using Franz diffusion cells was completed as previously described. ${ }^{6}$ The lower dorsal skin of C57BL/6 mice was used in all permeation studies. Hair was trimmed using an electric clipper 24 hours before collection of the skin, which was stored at $-20^{\circ} \mathrm{C}$ for a maximum period of one month and used when needed. After the fat layer was carefully removed, the skin was placed onto the flat surface of a balance, and the microneedle rollers were rolled in four perpendicular lines over the skin surface, five times each for a total of 20 times, with an applying pressure of 350 to $400 \mathrm{~g}$, which was constantly measured using the balance while rolling the microneedle roller. ${ }^{35}$ The skin was then mounted onto Franz diffusion cells from PermeGear Inc (Hellertown, PA), dorsal side facing upward. The receiver compartment contained $5 \mathrm{~mL}$ of phosphate-buffered saline ( $\mathrm{pH} 7.4,10 \mathrm{mM}$ ) and was maintained at $37^{\circ} \mathrm{C}$ with a Haake SC 100 water circulator from ThermoScientific (Wellington, NH). The diffusion area of the skin was $0.64 \mathrm{~cm}^{2}$. The donor compartment was loaded with fluorescein-labeled ovalbumin or fluorescein-ovalbumin nanoparticles in phosphate-buffered saline $(500 \mu \mathrm{L}, \mathrm{pH} 7.4,10 \mathrm{mM})$ and covered with parafilm to prevent evaporation. The amount of ovalbumin protein loaded into the donor compartment was $0.6 \mathrm{mg}$. At hours $0,1,2,4,8$, and $24,200 \mu \mathrm{L}$ samples were withdrawn from the receiver compartment and immediately replenished with fresh phosphate-buffered saline. The fluorescence intensity in the sample was measured using a BioTek Synergy ${ }^{\mathrm{TM}}$ HT multimode microplate reader (Winooski, VT). 


\section{Methylene blue staining for visualization of micropores}

Hair on the dorsal skin of C57BL/6 mice was trimmed before the mice were euthanized to remove the skin. The skin sample was treated with Nair ${ }^{\circledR}$ lotion (Church and Dwight Co, Princeton, NJ), rinsed with water, paper dried, and placed onto the flat surface of a balance. Microneedle rollers were rolled once over the skin surface with an applying pressure of 350 to $400 \mathrm{~g}$. The skin was then stained with $20 \mu \mathrm{L}$ of methylene blue solution for no more than five minutes, followed by removal of excessive stain using normal saline swabs and later alcohol swabs. Stained skin was visualized using a Stereoscopic Zoom Nikon SMZ1500 microscope (Melville, NY). As a control, skin was also punctured with a 21 gauge hypodermic needle (Becton Dickinson, Franklin Lakes, NJ).

\section{Immunization studies}

All animal studies were carried out following the National Institutes of Health guidelines for animal care and use. The animal protocol was approved by the Institutional Animal Care and Use Committee at The University of Texas at Austin. Female C57BL/6 mice (18-20 g) were used for the immunization studies. Twenty-four hours prior to the application of the vaccine formulations, hair on the dorsal side of the mice was carefully trimmed. The skin was cleaned with an alcohol swab, and a $2 \mathrm{~cm}^{2}$ area was marked on the skin surface. Mice were anesthetized and placed onto the flat surface of a balance to monitor the pressure applied during application of the microneedle rollers. The microneedle rollers were disinfected with ethanol $70 \%$ and then rolled in two perpendicular lines over the lower dorsal marked skin surface, ten times each, again for a total of 20 times, ${ }^{5}$ with an applying pressure of 350 to $400 \mathrm{~g}$. Ovalbumin in phosphatebuffered saline or ovalbumin-conjugated nanoparticles in phosphate-buffered saline were carefully dripped onto the treated area; the skin area was then covered with a piece of self-adhesive Tegaderm ${ }^{\circledR}$ patch (3M, St Paul, MN), which was carefully removed 24 hours later. Immunization was repeated 10 days apart on two further occasions. As a positive control, a group of mice were subcutaneously injected three times with ovalbumin-conjugated nanoparticles in phosphate-buffered saline. Two weeks (or as where mentioned) after the last immunization, mice were bled for antibody assay. The dose of ovalbumin was $10.5 \mu \mathrm{g}$ or $70 \mu \mathrm{g}$ per mouse.

\section{Enzyme-linked immunosorbent assay}

An enzyme-linked immunosorbent assay was completed as previously described. ${ }^{27,28}$ Briefly, EIA/RIA flat-bottomed, medium-binding, 96-well polystyrene plates (Corning Costar, Corning, NY) were coated with 100 ng of ovalbumin in $100 \mu \mathrm{L}$ of carbonate buffer $(10 \mathrm{mM}, \mathrm{pH} 9.6)$ overnight at $4{ }^{\circ} \mathrm{C}$. Plates were washed with phosphate-buffered saline/ Tween 20 (10 mM, pH 7.4, 0.05\% Tween 20, Sigma-Aldrich) and blocked with $5 \%(\mathrm{v} / \mathrm{v})$ horse serum in phosphatebuffered saline/Tween 20 for one hour at $37^{\circ} \mathrm{C}$. Samples were diluted 10 -fold serially in $5 \%(\mathrm{v} / \mathrm{v})$ horse serum in phosphate-buffered saline/Tween 20 , added to the plates following the removal of the blocking solution, and incubated for a further two hours at $37^{\circ} \mathrm{C}$. The serum samples were removed, and the plates were washed five times with phosphate-buffered saline/Tween 20. Horseradish peroxidase-labeled goat antimouse immunoglobulin G (5000-fold dilution in $1.25 \%(\mathrm{v} / \mathrm{v})$ horse serum in phosphate-buffered saline/Tween 20) was added into the plates, followed by another hour of incubation at $37^{\circ} \mathrm{C}$. Plates were again washed five times with phosphate-buffered saline/Tween 20. The presence of bound antibody was detected following incubation for 30 minutes at room temperature in the presence of 3,3',5,5'-tetramethylbenzidine solution, followed by addition of $0.2 \mathrm{M}$ sulfuric acid as the stop solution. The absorbance was read at $450 \mathrm{~nm}$ using a BioTek Synergy ${ }^{\mathrm{TM}}$ HT multimode microplate reader.

\section{Transepidermal water loss}

Mice were anesthetized, and hair on the lower dorsal skin was trimmed. Twenty-four hours later, the trimmed area was disinfected with ethanol $70 \%$ and treated with the microneedle rollers as mentioned earlier. Negative control mice received hair trimming only. Before and immediately after the needle treatment ( 0 hour), transepidermal water loss was measured using a VapoMeter from Delfin Technologies Inc (Stamford, CT) following the manufacturer's instructions. At least three readings were taken at every time point. If there were any uncharacteristic spikes during this period, a more representative reading was used. Transepidermal water loss readings were also recorded at hours 2,3 , 4, and 24. For mice treated with the large microneedle roller, transepidermal water loss readings were also recorded 48 hours after treatment. The experiment was repeated using at least four mice per group.

\section{In vitro permeation of bacteria through microneedle-treated skin}

Hair-trimmed mice were treated with the microneedle rollers on the lower dorsal skin (10 times each in two perpendicular directions, for a total of 20 times) and then immediately 
euthanized. The skin in the treated area was collected and used to evaluate the permeation of live bacteria on the same day. As controls, intact skin (hair trimmed) or skin punctured once with a 21 gauge needle were also used. In addition, for the microneedle roller with large microneedles, mice were treated with the roller and euthanized immediately or at hours $1,3,6$, or 24 to collect the skin in the treated area. The collected skin was mounted onto Franz diffusion cells to evaluate the microbial permeation. Mouse skin in the treated area and the working surface in a laminar flow cabinet were disinfected with ethanol $70 \%$ before treatment. All dissecting tools were autoclaved before use.

E. coli DH5 $\alpha$ bacteria were used to evaluate permeation of live bacteria through the treated skin. Bacteria were grown in Luria-Bertani medium (Sigma-Aldrich), harvested, and resuspended into the same volume of sterile phosphatebuffered saline $(\mathrm{pH} 7.4,10 \mathrm{mM})$. The $\mathrm{OD}_{600}$ value of the suspension was determined to be $1.27 \pm 0.11$. The bacterial suspension was diluted in sterile phosphate-buffered saline (pH 7.4, $10 \mathrm{mM}$ ) to 1000 -fold, and $500 \mu \mathrm{L}$ was then placed into the donor compartment of the diffusion cells. Four hours later, the sample in the receiver compartment was withdrawn, diluted 1-fold, 10-fold, and 100-fold in sterile phosphatebuffered saline, and $50 \mu \mathrm{L}$ was then spread onto Luria-Bertani agar plates, which were incubated at $37^{\circ} \mathrm{C}$ overnight to count the number of colonies formed. The number of bacteria diffused through the skin was reported as colony forming units, and it was assumed that each colony had developed from a single bacterial cell. The diffusion cells and the parafilm used to cover the cells were thoroughly disinfected with ethanol $70 \%$ three times before use, and all other items were autoclaved before use.

\section{Statistical analysis}

Statistical analyses were performed using analysis of variance followed by Fisher's protected least significant difference procedure. A $P$ value of $\leq 0.05$ (two-tailed) was considered statistically significant.

\section{Results and discussion}

The ovalbumin nanoparticles were $230 \pm 22 \mathrm{~nm}$ in diameter, with a polydispersity index of 0.2 . Their zeta potential was $-31 \pm 1 \mathrm{mV}$. The amount of ovalbumin conjugated onto the nanoparticles was determined to be $96.6 \pm 11.0 \mu \mathrm{g}$ ovalbumin per mg of nanoparticles. ${ }^{29}$ Lower dorsal mouse skin samples were harvested, treated with microneedle rollers, and used to evaluate permeation of the ovalbumin nanoparticles. Microscopic pictures of the skin stained with methylene blue solution immediately following treatment with different microneedle rollers are shown in Figure 2. As a control, the picture of the skin punctured by a 21 gauge hypodermic needle is also shown (Figure 2A). The single pore created by the hypodermic needle was about $1 \mathrm{~mm}$ in diameter, which is to be expected because the nominal outer diameter of a 21 gauge needle is $819.2 \mu \mathrm{m}$. The pores created by the microneedles were much smaller, and it seemed that the diameter of the micropores created using a roller with larger microneedles tended to be larger than that created using a roller with smaller microneedles (Figure 2), in agreement with what was previously reported by Zhou et al, ${ }^{5}$ who used ZTGS $^{\mathrm{TM}}$ microneedle rollers. Due to the extensive diffusion of the blue dye, an accurate measurement of the diameters of those micropores was not attempted.

As shown in Figures $3 \mathrm{~A}$ and $3 \mathrm{~B}$, neither ovalbumin protein in solution nor ovalbumin conjugated onto nanoparticles could permeate through the intact skin, demonstrating the physical integrity of the skin samples. In contrast, both ovalbumin and ovalbumin nanoparticles were able to permeate through skin pretreated with microneedle rollers (Figures 3A and 3B). Moreover, pretreatment using a roller with larger microneedles allowed more extensive permeation than treatment using a roller with smaller microneedles. For example, within 24 hours, only a minimum amount of ovalbumin nanoparticles permeated through the skin pretreated using a roller with small microneedles (200 $\mu \mathrm{m}$ long, base diameter $20 \mu \mathrm{m}$ ), whereas $13.6 \pm 2.4 \%$ of the ovalbumin nanoparticles permeated through the skin treated with the roller with large microneedles $(1000 \mu \mathrm{m}$ long, base diameter

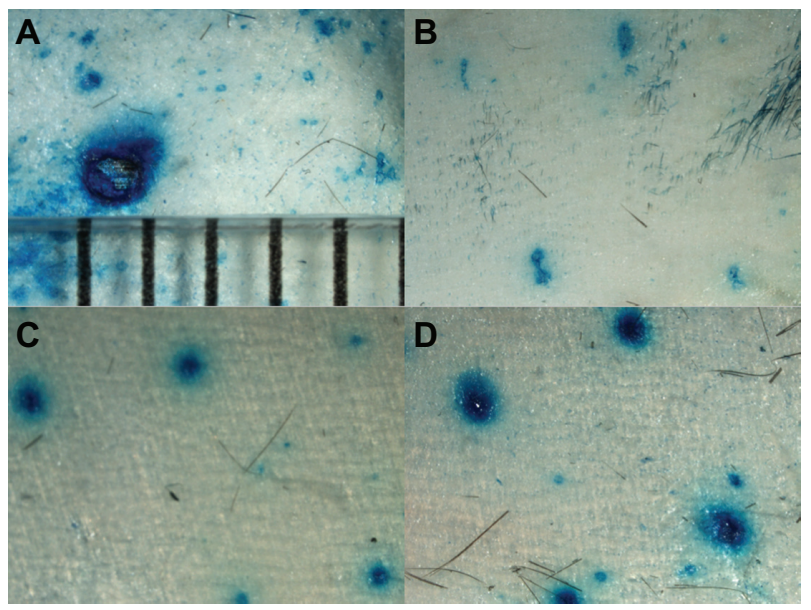

Figure 2 Magnified microscopic view of mouse skin after treatment with a 21 gauge hypodermic needle (A) or microneedle rollers with different size microneedles, ie, small (B), medium (C), and large (D). The skin was stained with methylene blue solution. Notes: The distance between the bars in $\mathbf{A}$ is I $\mathrm{mm}$; all photos were taken under the same magnification. 

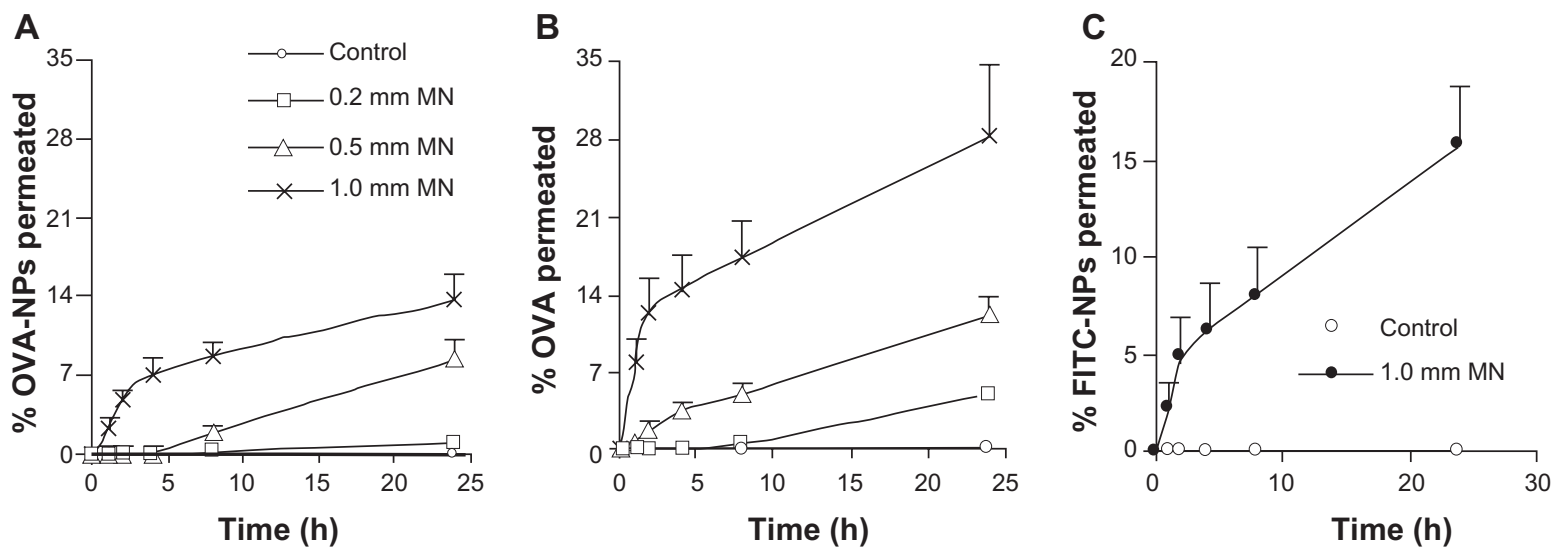

Figure 3 Permeation of fluorescein-labeled ovalbumin nanoparticles (A), fluorescein-labeled ovalbumin (B), or fluorescein-labeled ovalbumin-free nanoparticles (C) through mouse skin treated with different microneedles (small $200 \mu \mathrm{m}$, medium $500 \mu \mathrm{m}$, and large $1000 \mu \mathrm{m}$ ).

Note: Data shown are mean \pm standard error $(n=5-7)$.

Abbreviations: MN, microneedle; OVA, ovalbumin; NPs, nanoparticles; FITC, fluorescein-5(6)-isothiocyanate.

of $80 \mu \mathrm{m}$, Figure 3A). As expected, pretreatment with the microneedle rollers allowed more extensive permeation of the ovalbumin in solution than the ovalbumin conjugated onto nanoparticles (Figure 3A and 3B), considering that the ovalbumin nanoparticles are much larger than the ovalbumin molecules. For example, within 24 hours, $28.3 \pm 6.5 \%$ of the ovalbumin in solution diffused through the pores created by the roller with large microneedles, which is significantly higher than the $13.6 \pm 2.4 \%$ for the ovalbumin nanoparticles. To confirm that it was the ovalbumin nanoparticles, rather than the ovalbumin protein hydrolyzed from the ovalbumin nanoparticles, that diffused through the pores created by the microneedle rollers, permeation of the fluorescein-labeled nanoparticles alone was also monitored. As shown in Figure $3 \mathrm{C}$, the rate of diffusion of the fluorescein nanoparticles was similar to the diffusion of the fluorescein-labeled ovalbumin nanoparticles. Finally, diffusion of DOPEfluorescein from the fluorescein-labeled nanoparticles placed into a dialysis tube (molecular weight cut off, 50,000) was evaluated as well. It was found that, within 24 hours, release of DOPE-fluorescein from the nanoparticles was not detectable, regardless of whether the release medium was phosphate-buffered saline or phosphate-buffered saline with sodium dodecyl sulfate $0.5 \%$ (data not shown), which indicated that the observed permeation of fluorescein-labeled nanoparticles in Figure 3C was not caused by the diffusion of the DOPE-fluorescein molecules from the fluorescein-labeled nanoparticles and then through the skin.

Taken together, the data in Figure 3 demonstrate that ovalbumin nanoparticles of $230 \pm 22 \mathrm{~nm}$ permeated through the micropores created by a microneedle, even using the roller with the smallest microneedles $(200 \mu \mathrm{m}$ long, base diameter $20 \mu \mathrm{m})$, and that as expected, the extent of permeation was dependent on the size of the microneedles used. This observation is in agreement with that of Coulman et al who showed permeation of $138 \pm 22 \mathrm{~nm}$ polystyrene nanoparticles through human skin pretreated with microneedles ( $280 \mu \mathrm{m}$ long, base diameter $200 \mu \mathrm{m}){ }^{7}$ but is in disagreement with the reports by Zhang et al and Bal et al using poly(lactic-co-glycolic) acid nanoparticles $(166,206$, or $288 \mathrm{~nm})$ and diphtheria toxoid-N-trimethyl chitosan nanoparticles $\left(211 \pm 4 \mathrm{~nm}\right.$ ), respectively. ${ }^{8,25}$ In the study by $\mathrm{Bal}$ et al, the length of microneedles used was $300 \mu \mathrm{m} .{ }^{25}$ The size of the nanoparticles used in the present study was similar to that used by Bal et al. It is interesting that the ovalbumin nanoparticles permeated through the skin area pretreated with the smallest microneedles ( $200 \mu \mathrm{m}$ long, base diameter $20 \mu \mathrm{m}$ ). It is speculated that, besides particle size, other factors, such as materials used to prepare the nanoparticles, surface charge of the nanoparticles, and the strain and source of animals used to harvest skin all contributed to different observations in the different studies.

Finally, in the present study, for easy detection, diffusion of the fluorescein-labeled nanoparticles through the skin and into the receiver compartment was measured. Figure 3 clearly shows that the ovalbumin nanoparticles diffused into the receiver compartment. We are aware that, for transcutaneous immunization, one expects to target the antigen inside the skin, particularly the epidermis, not necessarily to deliver the antigen through the skin because the skin epidermis has abundant antigen-presenting cells. ${ }^{36}$

As shown in Figure 4A, both ovalbumin in solution or ovalbumin nanoparticles failed to induce an antiovalbumin immunoglobulin $\mathrm{G}$ response when applied to intact mouse skin with hair trimmed. However, pretreatment using the 
A

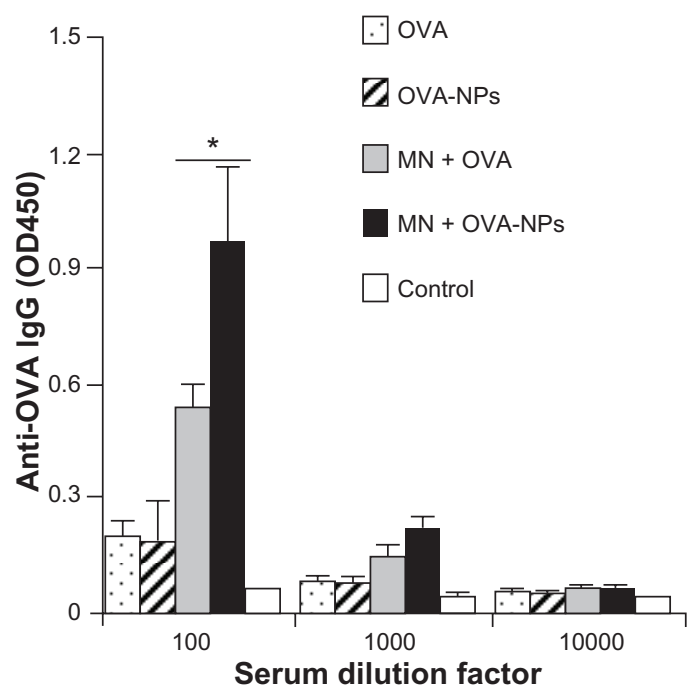

B

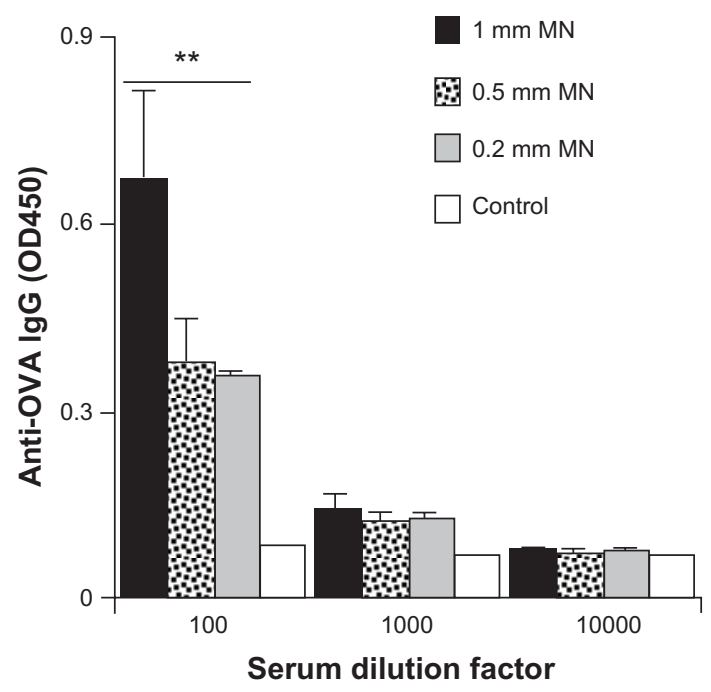

Figure 4 (A) Serum antiovalbumin immunoglobulin $\mathrm{G}$ induced by ovalbumin or ovalbumin nanoparticles applied onto a skin area treated or not treated with a microneedle roller (1000 $\mu \mathrm{m}$ long, base diameter $80 \mu \mathrm{m})$. (B) Antiovalbumin immunoglobulin $\mathrm{G}$ induced by ovalbumin nanoparticles applied onto a skin area treated with different microneedles. Data reported are mean \pm standard error for five mice per group. The ovalbumin dose was $70 \mu g$ per mouse.

Notes: Data reported in $(\mathbf{B})$ are one week after the last immunization; $* P=0.03$, microneedle roller treatment + ovalbumin versus microneedle roller treatment + ovalbumin nanoparticles; $* * P=0.000$ I, analysis of variance of the three different microneedles.

Abbreviations: MN, microneedle roller treatment; OVA, ovalbumin; NPs, nanoparticles; lgG, immunoglobulin G; OD, optical density.

microneedle roller with large microneedles $(1000 \mu \mathrm{m}$ long, base diameter $80 \mu \mathrm{m}$ ) allowed both ovalbumin alone and ovalbumin nanoparticles to induce an antiovalbumin immunoglobulin G response (Figure 4A). Importantly, the antiovalbumin immunoglobulin $\mathrm{G}$ level in mice that received the ovalbumin nanoparticles was significantly higher than that in mice that received ovalbumin alone (Figure 4A), demonstrating that, when dosed onto a mouse skin area pretreated with microneedles, formulating a protein antigen into nanoparticles can enhance its immunogenicity.

Bal et al showed that microneedle-mediated delivery of diphtheria toxoid incorporated into $\mathrm{N}$-trimethyl chitosan nanoparticles did not induce a stronger antibody response than the diphtheria toxoid alone. ${ }^{25}$ Therefore, it does not appear that formulating any protein antigen in any nanoparticles will be beneficial. Many factors, including the physical, chemical, and immunological properties of the nanoparticles, the antigen itself, and the dimension of the microneedles, may be responsible for the disagreement observed. Interestingly, Bal et al actually reported that when diphtheria toxoid was physically mixed with the N-trimethyl chitosan nanoparticles and applied onto mouse skin pretreated with microneedles, it induced a stronger antidiphtheria toxoid immune response than diphtheria toxoid alone. ${ }^{25}$ This observation led the authors to predict that conjugation of antigen with polymeric nanoparticles, instead of incorporation of antigens inside nanoparticles, could be a better option to potentiate further the immune responses by microneedle-mediated vaccination. ${ }^{25}$ Our data in Figure 4A appear to support their prediction. Therefore, more research on formulating the antigen of interest into the proper nanoparticles is warranted for successful microneedle-mediated immunization using antigens carried by nanoparticles.

Figure 4B shows the antiovalbumin immunoglobulin $\mathrm{G}$ response induced by the ovalbumin nanoparticles applied onto a mouse skin area pretreated with different sized microneedle rollers. As expected, pretreatment using the roller with large microneedles enabled induction of a significantly stronger antiovalbumin immunoglobulin $\mathrm{G}$ response than using the rollers with small and medium microneedles (Figure 4B). However, pretreatment using the roller with small microneedles and the roller with medium microneedles did not lead to different levels of antiovalbumin immunoglobulin $\mathrm{G}$ response (Figure 4B). The in vitro diffusion data in Figure 3A show that the roller with medium microneedles $(500 \mu \mathrm{m}$ long, base diameter $50 \mu \mathrm{m}$ ) allowed significantly more permeation of ovalbumin nanoparticles than the roller with small microneedles $(200 \mu \mathrm{m}$ long, base diameter $20 \mu \mathrm{m})$. It is possible that the amounts of ovalbumin nanoparticles that can permeate through the micropores created by these two different sized microneedles in vivo were not different enough to be detected by measuring the resulting antiovalbumin antibody levels. Therefore, it is likely that for any specific nanoparticle formulation, the optimal dimension of the microneedles to be used needs to be identified individually. 
Mice were treated with ovalbumin nanoparticles containing $10.5 \mu \mathrm{g}$ of ovalbumin initially in order to compare the antibody responses induced by ovalbumin nanoparticles applied onto a skin area pretreated using microneedles with the same ovalbumin nanoparticles applied by subcutaneous injection. As shown in Figure 5A, antiovalbumin immunoglobulin $\mathrm{G}$ levels induced by ovalbumin nanoparticles given by subcutaneous injection or by transcutaneous immunization following microneedle treatment were not significantly different $(P=0.38,100$-fold dilution). Moreover, it appeared that the antibody response induced by the ovalbumin nanoparticles dosed onto a skin area pretreated with microneedles was dose-dependent. For example, ovalbumin nanoparticles at a dose of $70 \mu \mathrm{g}$ per mouse applied onto a skin area pretreated with the microneedles induced a stronger antiovalbumin immunoglobulin $\mathrm{G}$ response than at a dose of $10.5 \mu \mathrm{g}$ (Figure 5A). However, when the ovalbumin dose was increased from $10.5 \mu \mathrm{g}$ per mouse to $70 \mu \mathrm{g}$ per mouse, transcutaneous immunization following microneedle treatment induced a weaker antiovalbumin immunoglobulin $\mathrm{G}$ response than subcutaneous injection (Figure 5B), indicating that the antigen dose determines whether transcutaneous immunization following microneedle treatment with antigens carried by nanoparticles is more effective than subcutaneous injection. The dose of $70 \mu \mathrm{g}$ ovalbumin per mouse was initially selected because data from a previous study showed that subcutaneous immunization with $70 \mu \mathrm{g}$ of ovalbumin in ovalbumin-conjugated nanoparticles induced a strong antibody response..$^{29}$ The ovalbumin dose of $10.5 \mu \mathrm{g}$ (ie, $15 \%$ of $70 \mu \mathrm{g}$ ) per mouse was used later because the in vitro data in Figure 3A showed that, within 24 hours, only about $15 \%$ of the ovalbumin nanoparticles permeated through a mouse skin area pretreated using the roller with large microneedles. Of course it is likely that in vivo, less than $15 \%$ of the ovalbumin nanoparticles have permeated through the skin treated using the same microneedle roller due to factors such as accelerated closure of the micropores and less than ideal permeation conditions. Moreover, it is known that microneedle puncture is less efficient in vivo than in vitro because of the more flexible skin tissue, a nonflat skin surface, the cushioning effect of fat and muscle layers. ${ }^{37}$ Nonetheless, transcutaneous immunization with a nanoparticle-based vaccine formulation onto a skin area pretreated with microneedles has the potential to elicit a stronger immune response than that achieved by subcutaneous injection using a hypodermic needle.

Transepidermal water loss was measured to evaluate the extent to which treatment with microneedle rollers had damaged the integrity of the skin. As shown in Figure 6, transepidermal water loss in the treated skin area increased significantly, and the roller with larger microneedles led to a larger increase in transepidermal water loss immediately after treatment using the microneedle rollers. Transepidermal water loss then gradually decreased and reached a level similar to that of intact skin within 24 hours when the rollers with small and medium microneedles were used (Figure 6), in agreement with what was previously reported. ${ }^{5}$ However, it
A

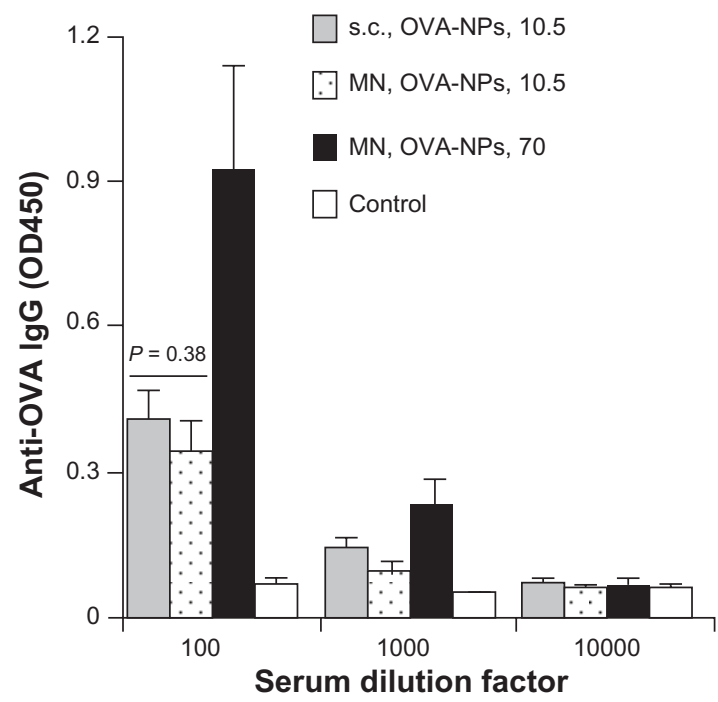

B

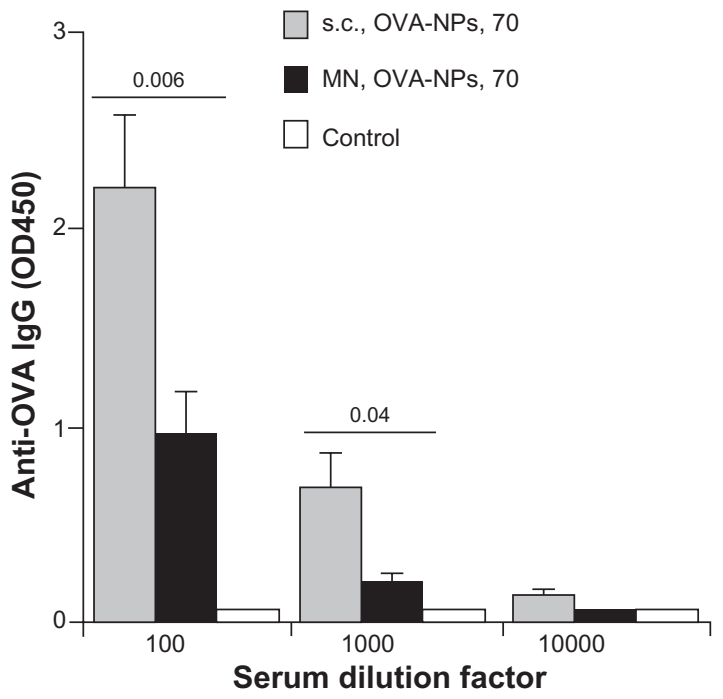

Figure $\mathbf{5}$ The effect of dose of ovalbumin as an antigen on the antibody responses induced by the ovalbumin nanoparticles. Shown are antiovalbumin immunoglobulin $\mathrm{G}$ when the dose of the ovalbumin in the ovalbumin nanoparticles was $10.5 \mu \mathrm{g}$ per mouse (A) or $70 \mu \mathrm{g}$ per mouse (B).

Notes: The microneedle roller used was the one with large microneedles; data shown are mean \pm standard error of the mean from five mice per group.

Abbreviations: MN, microneedle roller treatment; OVA, ovalbumin; NPs, nanoparticles; IgG, immunoglobulin G. 


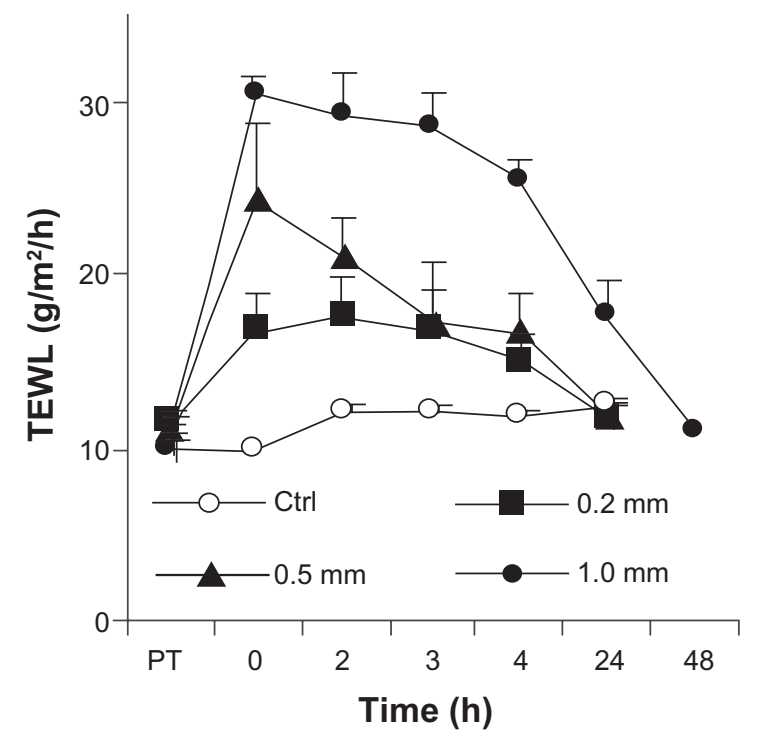

Figure 6 Transepidermal water loss from the skin at different time points after treatment with different microneedles.

Note: Data shown are mean \pm standard error of the mean $(n=4)$

Abbreviations: TEWL, transepidermal water loss; PT, prior to treatment with microneedles; ctrl, control.

took a longer period of time, ie, 48 hours, for transepidermal water loss on the skin area pretreated using the roller with large microneedles $(1000 \mu \mathrm{m}$ long, base diameter $80 \mu \mathrm{m})$ to reach the intact level (Figure 6).

The kinetics of transepidermal water loss in Figure 6 confirmed that treatment with microneedles caused physical damage, albeit reversible, to the skin, which was previously known. ${ }^{5}$ However, the relevance of this reversible physical damage is not well understood. Specifically, it is unknown to what extent the micropores created by the microneedles may enhance penetration of microbes through the treated skin area, considering that microbes, such as bacteria and viruses, exist physically as nanoparticles or microparticles in the environment and on the skin surface. This information is clinically relevant because it allows prediction of the potential risk or lack of risk of microbial infection associated with treatment using microneedles. Recognizing this issue, Donnelly et al studied in vitro permeation of radiolabeled microbes through porcine skin pretreated with microneedles. ${ }^{34}$ In their study, harvested porcine skin was saturated with bacteria and then treated with microneedles to evaluate the extent to which the microneedles can carry pre-existing microbes through the skin. ${ }^{34}$ In the present study, an ex vivo system was devised to evaluate the extent to which pre-existing micropores created by the microneedles will allow permeation of live bacteria through the skin. Anesthetized mice were treated with the microneedle rollers and immediately euthanized to harvest the treated skin samples, which were then used to evaluate permeation of live nonpathogenic E. coli DH5 $\alpha$. E. coli is a rod-shaped bacterium about $200-500 \mathrm{~nm}$ in diameter and $2 \mu \mathrm{m}$ long, which is physically a nanorod particle. ${ }^{38}$ As shown in Figure 7A, live E. coli DH5 $\alpha$ bacteria can permeate through micropores created by microneedle rollers on the skin, and pretreatment using a roller with larger microneedles allowed permeation of more bacteria. It was determined that using the present method, the microneedle rollers created about 250 pores $/ \mathrm{cm}^{2}$ on the treated skin area. The area in the Franz diffusion cells was $0.64 \mathrm{~cm}^{2}$, which means that the number of bacterial colony forming units shown in Figure 7A represent the total number of bacteria that permeated through roughly 160 micropores created by the microneedle rollers within four hours. Data in Figure 7B showed that the micropores created by the microneedles also closed rather quickly. Within 3-6 hours of microneedle treatment, the pores became impermeable to $E$. coli bacteria, in agreement with what was previously reported, ie, that skin recovers its barrier function 3-4 hours after microneedle treatment. ${ }^{39}$

Data in Figure 7A indicate that the number of E. coli bacteria permeating through the single pore created by a 21 gauge hypodermic needle within four hours was equal to the number of $E$. coli permeating through the micropores (about 160) created by the roller with large microneedles $(1000 \mu \mathrm{m}$ long, base diameter $80 \mu \mathrm{m})$ within the same period of time. In other words, one single pore created by the 21 gauge hypodermic needle was equivalent to about 160 micropores created by the roller with large microneedles. Clinically, a 21 gauge needle is normally used to withdraw blood, and smaller needles are generally used for vaccination. It is expected that the risk of bacterial infection associated with microneedle treatment is more likely to be less than the risk associated with a hypodermic needle injection. Nonetheless, the finding in the present study does underscore the need for sterilization of any formulation that is to be applied onto a skin area pretreated with microneedles and also the need to keep the application area clean prior to and after microneedle treatment. Of course, the microneedles per se should be pathogen-free as well.

All the aforementioned experiments were carried out using C57BL/6 mice and their skin. It is known that human skin is significantly thicker than mouse skin. Therefore, any findings made in a mouse model will ultimately need to be validated in humans. Before transition to humans, porcine skin is a good model to predict more accurately what is expected in humans because porcine skin is very similar to human skin. ${ }^{40}$ 
A

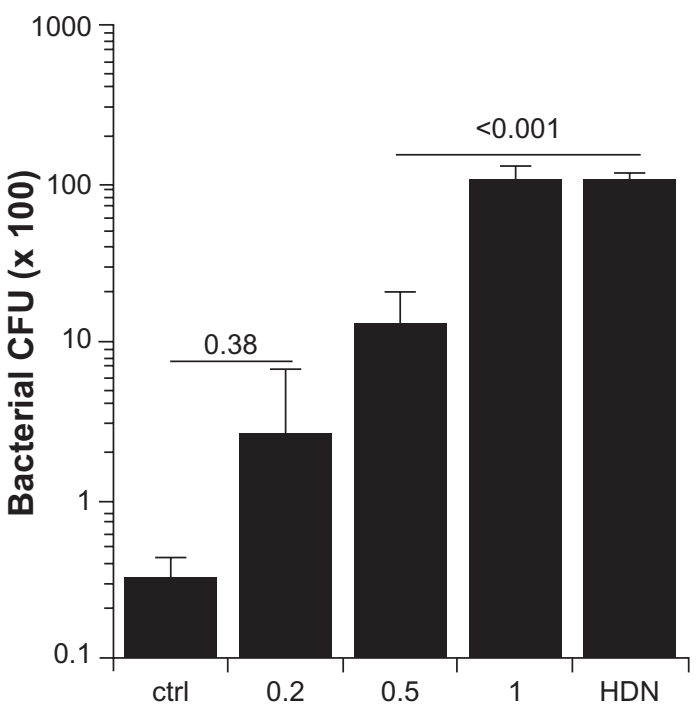

B

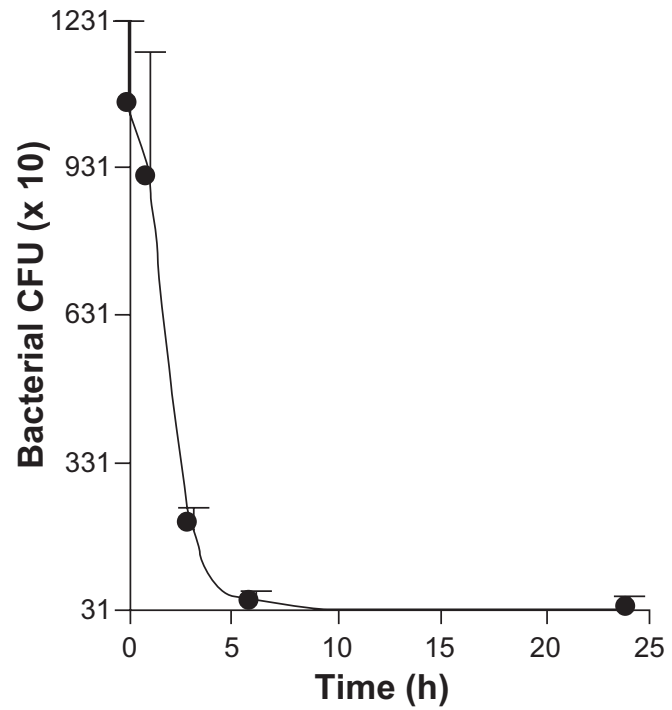

Figure 7 Permeation of Escherichia coli DH5 $\alpha$ through skin treated with microneedle rollers. (A) Number of bacteria, indicated by bacterial colony forming units, permeated through the micropores created by different microneedles on an area of $0.64 \mathrm{~cm}^{2}$. As controls, intact skin or skin with a single pore created by a 21 gauge hypodermic needle were used. (B) Number of bacteria (colony forming units) permeated through micropores created by the roller with large microneedles at different times after microneedle treatment. Notes: All numbers were after four hours of permeation; data shown are mean \pm standard deviation $(n=3)$.

Abbreviations: CFU, colony forming units; ctrl, control; HDN, hypodermic needle.

Finally, microneedles have been exploited in various ways to deliver vaccine, ie, solid microneedles coated with vaccines, dissolvable microneedles with vaccine incorporated in the needles, hollow microneedle-based injection, and the application of a vaccine formulation onto the skin prior to or after the skin area was treated with microneedles. At this moment, transcutaneous immunization on a skin area pretreated with microneedles has the slight limitation of being a two-step procedure. However, it is not impossible that this limitation can be overcome by creative engineering in the future. Moreover, all the four methods mentioned above have their own unique advantages and disadvantages. ${ }^{26}$ Solid microneedles of sufficient strength are commercially available, and it is economical to mass produce them. However, coating of a particular vaccine onto solid microneedles involves reformulation to optimize viscosity and protein concentration to avoid aggregation. ${ }^{26}$ The long-term stability of a dry-coated microneedle vaccine is likely better than for liquid injectables, but the stability of a particular vaccine is dependent on refined formulation and appropriate packaging. ${ }^{24}$ In addition to all these issues, immunization via a coated solid microneedle is also a multistep process. Immunization needs administration of needles, a waiting time of 1-2 minutes to allow the coating to dissolve and, finally, application of a patch over the treated area. The manufacturing of dissolvable microneedles with sufficient strength is still a challenge, and laboratory scale production of dissolvable microneedles usually involves the melting of polymers at a high temperature, which is detrimental to protein stability. ${ }^{41}$ Hollow microneedles for injection suffer from concerns about potential clogging, back pressure from densely packed skin layers, and aggregation and syringeability for highly concentrated formulations. ${ }^{26}$ In addition, stability of the proteins and leakage issues during storage of prefilled hollow microneedles are still of practical concern. ${ }^{26}$ Therefore, the perceived inconvenience associated with the two-step procedure of transcutaneous immunization prior to or after microneedle treatment should not preclude further research efforts. Moreover, knowledge gleaned from using solid microneedles is always transferable to other microneedle systems.

\section{Conclusion}

Pretreatment with microneedles allowed skin permeation of nanoparticles with antigen protein conjugated onto them. Transcutaneous immunization onto a skin area pretreated with microneedles with the protein antigen carried by nanoparticles induced a stronger antigen-specific antibody response than using the protein antigen alone. The antigen dose used to immunize the mice determined whether the microneedle-mediated immunization can induce a stronger immune response than when the same nanoparticle-based vaccine formulation was dosed by subcutaneous injection. Damage to the physical integrity of the skin caused by 
microneedles, although reversible, may permit permeation of live bacteria through the skin, but the risk of bacterial infection associated with microneedles is not expected to be higher than that associated with injection using a hypodermic needle. With the increasing interest in nanoparticles as a drug delivery system, more research on skin permeation of nanoparticles prior to or after microneedle treatment is warranted.

\section{Acknowledgment}

This work was supported in part by National Institute of Allergy and Infectious Diseases grants (AI078304 and $\mathrm{AI} 070538$ ) to ZC.

\section{Disclosure}

The authors report no conflicts of interest in this work.

\section{References}

1. Prausnitz MR. Microneedles for transdermal drug delivery. Adv Drug Deliv Rev. 2004;56:581-587.

2. Park JH, Choi SO, Seo S, Choy YB, Prausnitz MR. A microneedle roller for transdermal drug delivery. Eur J Pharm Biopharm. 2010;76: 282-289.

3. Yoo KH, Lee JW, Li K, Kim BJ, Kim MN. Photodynamic therapy with methyl 5-aminolevulinate acid might be ineffective in recalcitrant alopecia totalis regardless of using a microneedle roller to increase skin penetration. Dermatol Surg. 2010;36:618-622.

4. You SK, Noh YW, Park HH, et al. Effect of applying modes of the polymer microneedle-roller on the permeation of L-ascorbic acid in rats. J Drug Target. 2010;18:15-20.

5. Zhou CP, Liu YL, Wang HL, Zhang PX, Zhang JL. Transdermal delivery of insulin using microneedle rollers in vivo. Int J Pharm. 2010;392: 127-133.

6. McAllister DV, Wang PM, Davis SP, et al. Microfabricated needles for transdermal delivery of macromolecules and nanoparticles: Fabrication methods and transport studies. Proc Natl Acad Sci U S A. 2003;100: 13755-13760.

7. Coulman SA, Anstey A, Gateley C, et al. Microneedle mediated delivery of nanoparticles into human skin. Int J Pharm. 2009;366:190-200.

8. Zhang W, Gao J, Zhu Q, et al. Penetration and distribution of PLGA nanoparticles in the human skin treated with microneedles. Int J Pharm. 2010;402:205-212.

9. Oyewumi MO, Kumar A, Cui Z. Nano-microparticles as immune adjuvants: Correlating particle sizes and the resultant immune responses. Expert Rev Vaccines. 2010;9:1095-1107.

10. Prausnitz MR, Mikszta JA, Cormier M, Andrianov AK. Microneedlebased vaccines. Curr Top Microbiol Immunol. 2009;333:369-393.

11. Kim YC, Quan FS, Compans RW, Kang SM, Prausnitz MR. Formulation of microneedles coated with influenza virus-like particle vaccine. AAPS Pharm Sci Tech. 2010;11:1193-1201.

12. Kim YC, Quan FS, Compans RW, Kang SM, Prausnitz MR. Formulation and coating of microneedles with inactivated influenza virus to improve vaccine stability and immunogenicity. $J$ Control Release. 2010;142:187-195.

13. Kim YC, Quan FS, Yoo DG, Compans RW, Kang SM, Prausnitz MR. Improved influenza vaccination in the skin using vaccine coated microneedles. Vaccine. 2009;27:6932-6938.

14. Kim YC, Quan FS, Yoo DG, Compans RW, Kang SM, Prausnitz MR. Enhanced memory responses to seasonal $\mathrm{H} 1 \mathrm{~N} 1$ influenza vaccination of the skin with the use of vaccine-coated microneedles. J Infect Dis. 2010;201:190-198.
15. Koutsonanos DG, del Pilar Martin M, Zarnitsyn VG, et al. Transdermal influenza immunization with vaccine-coated microneedle arrays. PLoS One. 2009;4:e4773.

16. Mikszta JA, Dekker JP 3rd, Harvey NG, et al. Microneedle-based intradermal delivery of the anthrax recombinant protective antigen vaccine. Infect Immun. 2006;74:6806-6810.

17. Prow TW, Chen X, Prow NA, et al. Nanopatch-targeted skin vaccination against West Nile virus and Chikungunya virus in mice. Small. 2010; 6:177-184.

18. Quan FS, Kim YC, Compans RW, Prausnitz MR, Kang SM. Dose sparing enabled by skin immunization with influenza virus-like particle vaccine using microneedles. J Control Release. 2010;147:326-332.

19. Quan FS, Kim YC, Vunnava A, et al. Intradermal vaccination with influenza virus-like particles by using microneedles induces protection superior to that with intramuscular immunization. J Virol. 2010; 84:7760-7769.

20. Quan FS, Kim YC, Yoo DG, Compans RW, Prausnitz MR, Kang SM. Stabilization of influenza vaccine enhances protection by microneedle delivery in the mouse skin. PLoS One. 2009;4:e7152.

21. Song JM, Kim YC, Barlow PG, et al. Improved protection against avian influenza $\mathrm{H} 5 \mathrm{~N} 1$ virus by a single vaccination with virus-like particles in skin using microneedles. Antiviral Res. 2010;88:244-247.

22. Song JM, Kim YC, Lipatov AS, et al. Microneedle delivery of H5N1 influenza virus-like particles to the skin induces long-lasting B- and T-cell responses in mice. Clin Vaccine Immunol. 2010;17:1381-1389.

23. Sullivan SP, Koutsonanos DG, Del Pilar Martin M, et al. Dissolving polymer microneedle patches for influenza vaccination. Nat Med.2010; 16:915-20.

24. Widera G, Johnson J, Kim L, et al. Effect of delivery parameters on immunization to ovalbumin following intracutaneous administration by a coated microneedle array patch system. Vaccine. 2006; 24:1653-1664.

25. Bal SM, Ding Z, Kersten GF, Jiskoot W, Bouwstra JA. Microneedlebased transcutaneous immunisation in mice with N-trimethyl chitosan adjuvanted diphtheria toxoid formulations. Pharm Res. 2010; 27:1837-1847.

26. Kalluri H, Banga AK. Transdermal delivery of proteins. AAPS Pharm Sci Tech. 2011;12:431-41.

27. Sloat BR, Sandoval MA, Hau AM, He Y, Cui Z. Strong antibody responses induced by protein antigens conjugated onto the surface of lecithin-based nanoparticles. J Control Release. 2010;141: 93-100.

28. Sloat BR, Sandoval MA, Cui Z. Towards preserving the immunogenicity of protein antigens carried by nanoparticles while avoiding the cold chain. Int J Pharm. 2010;393:197-202.

29. Li X, Sloat BR, Yanasarn N, Cui Z. Relationship between the size of nanoparticles and their adjuvant activity: Data from a study with an improved experimental design. Eur J Pharm Biopharm. 2011; 78:107-116.

30. Laurent PE, Bourhy H, Fantino M, Alchas P, Mikszta JA. Safety and efficacy of novel dermal and epidermal microneedle delivery systems for rabies vaccination in healthy adults. Vaccine. 2010;28: 5850-5856.

31. Van Damme P, Oosterhuis-Kafeja F, Van der Wielen M, Almagor Y, Sharon O, Levin Y. Safety and efficacy of a novel microneedle device for dose sparing intradermal influenza vaccination in healthy adults. Vaccine. 2009;27:454-459.

32. Bal SM, Caussin J, Pavel S, Bouwstra JA. In vivo assessment of safety of microneedle arrays in human skin. Eur J Pharm Sci. 2008; 35:193-202.

33. Gomaa YA, Morrow DI, Garland MJ, Donnelly RF, El-Khordagui LK, Meidan VM. Effects of microneedle length, density, insertion time and multiple applications on human skin barrier function: Assessments by transepidermal water loss. Toxicol In Vitro. 2010;24:1971-1978.

34. Donnelly RF, Singh TR, Tunney MM, et al. Microneedle arrays allow lower microbial penetration than hypodermic needles in vitro. Pharm Res. 2009;26:2513-2522. 
35. Li G, Badkar A, Kalluri H, Banga AK. Microchannels created by sugar and metal microneedles: Characterization by microscopy, macromolecular flux and other techniques. J Pharm Sci. 2010;99:1931-1941.

36. Cui Z, Dierling A, Foldvari M. Non-invasive immunization on the skin using DNA vaccine. Curr Drug Deliv. 2006;3:29-35.

37. Teo MA, Shearwood C, Ng KC, Lu J, Moochhala S. In vitro and in vivo characterization of MEMS microneedles. Biomed Microdevices. 2005;7:47-52.

38. Pierucci O. Dimensions of Escherichia coli at various growth rates: Model for envelope growth. J Bacteriol. 1978;135:559-574.
39. Kalluri H, Banga AK. Formation and closure of microchannels in skin following microporation. Pharm Res. 2011;28:82-94.

40. Barbero AM, Frasch HF. Pig and guinea pig skin as surrogates for human in vitro penetration studies: A quantitative review. Toxicol In Vitro. 2009;23:1-13.

41. Donnelly RF, Raj Singh TR, Woolfson AD. Microneedle-based drug delivery systems: Microfabrication, drug delivery, and safety. Drug Deliv. 2010;17:187-207.

\section{Publish your work in this journal}

The International Journal of Nanomedicine is an international, peerreviewed journal focusing on the application of nanotechnology in diagnostics, therapeutics, and drug delivery systems throughout the biomedical field. This journal is indexed on PubMed Central, MedLine, CAS, SciSearch ${ }^{\circledR}$, Current Contents ${ }^{\circledR} /$ Clinical Medicine,
Journal Citation Reports/Science Edition, EMBase, Scopus and the Elsevier Bibliographic databases. The manuscript management system is completely online and includes a very quick and fair peer-review system, which is all easy to use. Visit http://www.dovepress.com/ testimonials.php to read real quotes from published authors. 